\title{
Improved prognosis for acquired aplastic anaemia
}

\author{
L A Pitcher, I M Hann, J P M Evans, P Veys, J M Chessells, D K H Webb
}

\begin{abstract}
This study compared the prognosis of patients treated for aplastic anaemia at Great Ormond Street Hospital for Children from 1973-88 (group A; $n=38$ ) with a more recent cohort from 1989-96 (group $B ; \mathbf{n}=37)$. The two groups were similar in terms of clinical history, age, and severity of aplasia. The main treatment differences included the use of androgen treatment in group $A$ (21 of 38 patients) but not in group $B$, and the addition of cyclosporin A to immunosuppressive treatment for 14 patients in group B. Actuarial survival at eight years' follow up was significantly better for group B $(84 \% ; 95 \%$ CI, $68 \%$ to $93 \%$ ) than for group A $(45 \%$; $95 \%$ CI, $30 \%$ to $60 \%$ ), because of improved outcome for both immunosuppressive treatment $(86 \% v 39 \%)$ and bone marrow transplantation $(93 \% v 56 \%)$. There was no evidence for late clonal disorders or secondary malignancies in survivors in either group. The prognosis for aplastic anaemia has improved greatly in recent years so that over $80 \%$ of children are long term survivors.

(Arch Dis Child 1999;80:158-162)
\end{abstract}

Keywords: aplastic anaemia; immunosuppression; bone marrow transplantation; prognosis

Acquired aplastic anaemia (AA) in childhood is an uncommon but serious disorder, which affects approximately 2 in 1000000 children each year, and is characterised by pancytopenia as a result of marrow hypocellularity in the absence of a predisposing constitutional anomaly. ${ }^{1}$ The mechanisms for marrow failure are unclear, with evidence of both stem cell dysfunction and immune mediated ( $\mathrm{T}$ cell) suppression of haemopoiesis. ${ }^{2}$ Spontaneous recovery is rare, and survival with supportive care alone is poor, largely because of mortality from sepsis and haemorrhage. Bone marrow transplantation from a matched sibling donor, widely regarded as the treatment of choice, is possible in fewer than $30 \%$ of patients, ${ }^{3}$ and until recently, specific treatment with antilymphocyte globulin or androgens offered limited survival benefit compared with supportive care. ${ }^{4}$

In the last 15 years, new treatment strategies have been developed to improve the prognosis for children with acquired AA who do not have a matched sibling donor. These include the abandonment of androgen treatment in favour of immunosuppression, ${ }^{5}$ the use of cyclosporin A especially in combination with antilymphocyte globulin, ${ }^{6-8}$ the addition of haematopoietic growth factors to immunosuppressive regimens, ${ }^{9}$ and the increased consideration of matched unrelated donor stem cell transplantation where primary treatment has failed. ${ }^{10}$

We compared the outcome for children diagnosed and treated during the consecutive periods 1973-88 (group A) and 1989-96 (group B), to assess the impact of these changes on prognosis.

\section{Patients and methods}

PATIENTS

The outcome for children diagnosed with AA at Great Ormond Street Hospital for Children from 1973-88 (group A) has been reported. ${ }^{4}$ Data from this study were updated and a further case note review was conducted for all children diagnosed between January 1989 and December 1996 (group B). Patient characteristics recorded included clinical history, age, sex, severity of aplasia according to the modified Camitta criteria, ${ }^{2}$ and details of treatment including haematological response. Investigations in each patient included diagnostic bone marrow aspirate and trephine with cytogenetic analysis, a lymphocyte stress test with diepoxybutane or mitomycin $\mathrm{C}$ to exclude Fanconi's anaemia, and a Ham's test to exclude paroxysmal nocturnal haemoglobinuria. The type of bone marrow transplant as well as engraftment status, procedure related complications, and late effects were noted where appropriate.

\section{TREATMENT}

Group B children with severe disease and a matched sibling donor identified at diagnosis received a bone marrow transplant as initial treatment. Standard preparative treatment was with cyclophosphamide $(200 \mathrm{mg} / \mathrm{kg})$, with cyclosporin as graft versus host disease (GVHD) prophylaxis. Children without a matched sibling donor received immunosuppressive treatment either as antilymphocyte globulin alone $(15 \mathrm{mg} / \mathrm{kg} /$ day for five days) or combined with continuous cyclosporin A $(5-10 \mathrm{mg} / \mathrm{kg} /$ day; antilymphocyte globulin/cyclosporin A). All patients receiving immunosuppressive treatment were given steroid treatment (methylprednisolone $2-5 \mathrm{mg} / \mathrm{kg} /$ day) to prevent allergic reactions to antilymphocyte globulin. Children with an inadequate response after four months received rabbit antithymocyte globulin ( $3.75 \mathrm{mg} / \mathrm{kg} /$ day for five days). Cyclosporin A was continued and tapered to zero once a response (see below) was sustained with independence from blood and platelet transfusion. Unrelated donor bone marrow transplantation was considered for patients where immunosuppressive treatment had failed, or following relapse. The preparative regimen for these transplants included single fraction total 
Table 1 Comparison of clinical features of children with aplastic anaemia diagnosed during two time periods

\begin{tabular}{lll}
\hline & Group A (1973-88) & Group B (1989-96) \\
\hline Age (years) & $7(1-4)$ & $7(2-14)$ \\
Sex (male:female) & $17: 21$ & $23: 14$ \\
Severity (non-severe:severe:very severe) & $9: 16: 13$ & $9: 18: 10$ \\
Haemoglobin g/l median (range) & $74(30-110)$ & $75(24-109)$ \\
Platelets $\times 10^{9} / 1$ median (range) & $11(1-40)$ & $10(2-73)$ \\
Neutrophils $\times 10^{9} / 1$ median (range) & $0.4(0.1-2)$ & $0.5(0.1-1.5)$ \\
Reticulocytes $\%$ median (range) & $0.4(0.1-2.5)$ & $0.4(0.1-2)$ \\
\hline
\end{tabular}

body irradiation (300-400 cGy), cyclophosphamide $200 \mathrm{mg} / \mathrm{kg}$, and Campath $1 \mathrm{G} 1 \mathrm{mg} /$ $\mathrm{kg}$, with GVHD prophylaxis using cyclosporin $\mathrm{A}$ and short course methotrexate. Engraftment status was assessed using $\mathrm{X}-\mathrm{Y}$ chromosome fluorescent in situ hybridisation for sex mismatched bone marrow transplants, and by restriction fragment length polymorphism analysis for sex matched bone marrow transplants.

The treatment received by children in group A has been detailed previously. ${ }^{4}$ Matched sibling donor bone marrow transplantation commenced in 1978, and before 1980 most children were treated with oxymetholone $2.5 \mathrm{mg} / \mathrm{kg} /$ day, usually combined with prednisolone. After 1980, most children received antilymphocyte globulin alone (as above) or supplemented with a four month course of androgens. If the response was inadequate, a course of antithymocyte globulin was given, and matched unrelated donor bone marrow transplantation was considered.

RESPONSE ANALYSIS

A complete response was defined as the return of the blood count to normal for the child's age, a partial response as a lesser degree of improvement (haemoglobin $\geqslant 100 \mathrm{~g} / \mathrm{l}$; granulocytes $\geqslant 0.75 \times 10^{9} / 1$; platelets $\geqslant 50 \times$ $10^{9} / 1$ ), with independence from blood and platelet transfusions, and no response as failure to improve. ${ }^{6}$ Survival data are presented as Kaplan-Meier curves evaluated from the time of diagnosis up until the most recent review (October 1997) within the various treatment groups.

\section{Results}

PATIENT CHARACTERISTICS

Group A $(n=38)$ and group B $(n=37)$ were comparable in terms of age at presentation, and severity of aplasia (table 1). Group A contained fewer boys; however, this difference was not significant. The median duration of follow up was 180 months (range, 136-288) for group A and 57 months (range, 11-105) for group B.

TREATMENT

Table 2 summarises details of treatment received. One child in group A received supportive care only, 13 received androgens alone or in combination with prednisolone, 18 were given immunosuppressive treatment (11 antilymphocyte globulin only, seven antilymphocyte globulin in combination with androgens), and six received a matched sibling donor bone marrow transplant as initial treatment. Cyclosporin A was not used with antilym-
Table 2 Treatment of 75 children with acquired aplastic anaemia, 1973-96

\begin{tabular}{lll}
\hline Treatment & $\begin{array}{l}\text { Group A } \\
(1973-88) \\
\text { number (\%) }\end{array}$ & $\begin{array}{l}\text { Group B } \\
(1989-96) \\
\text { number (\%) }\end{array}$ \\
\hline None & $1(3)$ & $2(5)$ \\
Androgens & $13(34)$ & 0 \\
ALG \pm androgens & $18(47)$ & $11(30)$ \\
ALG/CSA & 0 & $14(38)$ \\
Initial BMT & $6(16)$ & $10(27)$ \\
Total & $38(100)$ & $37(100)$ \\
BMT after failed IS & 3 & 6 \\
\hline
\end{tabular}

$\overline{\mathrm{ALG} \text {, antilymphocyte globulin; CSA, cyclosporin; BMT, bone }}$ marrow transplantation; IS, immunosuppression.

phocyte globulin as initial treatment in this group. Three patients in group A received a matched unrelated donor bone marrow transplant after failed immunosuppressive treatment.

In group $\mathrm{B}$, two children received no treatment (one had early and spontaneous marrow recovery; the other died soon after diagnosis as a result of pseudomonas cellulitis and septicaemia), 25 received immunosuppressive treatment (11 antilymphocyte globulin only, 14 antilymphocyte globulin/cyclosporin A), and 10 had a matched sibling donor bone marrow transplant as initial treatment. Most (12 of 14) of the children treated with cyclosporin A received $5 \mathrm{mg} / \mathrm{kg} / \mathrm{day}$, and the remainder received $10 \mathrm{mg} / \mathrm{kg} /$ day. Nine children were given growth factor support in combination with immunosuppressive treatment (seven with granulocyte colony stimulating factor and two with granulocyte macrophage colony stimulating factor). Six children received a bone marrow transplant after failure of immunosuppressive treatment (two from matched unrelated donors, three from matched sibling donors, and one from a single antigen mismatch family donor). The patients given a matched sibling donor bone marrow transplant after failure of immunosuppressive treatment had non-severe disease at diagnosis.

\section{TREATMENT OUTCOME}

Actuarial survival after eight years was significantly better $(\mathrm{p}=0.001)$ for group B $(84 \%$; $95 \%$ CI, $68 \%$ to $93 \%)$ than group A (45\%; $95 \% \mathrm{CI}, 30 \%$ to $60 \%$ ). When the whole cohort was divided into three consecutive eight year time periods (fig 1), there was no significant difference in survival for patients in group A who were diagnosed between 1973-80 and 1981-88, while survival of patients in group B was significantly better $(p=0.005)$. This improvement was a result of a better outcome for both immunosuppressive treatment $(86 \% v$ $39 \%$; $=0.004$; fig 2 ) and bone marrow transplantation ( $93 \%$ v 56\%; $\mathrm{p}=0.04$; fig 3). When calculating survival of children treated by immunosuppressive treatment, patients were censored at the time of any subsequent bone marrow transplant (three group $\mathrm{A}$ and six group B patients). Survival of children given antilymphocyte globulin without cyclosporin A did not improve with time when patients from groups A and B were compared $(73 \%$ v $39 \%$; $\mathrm{p}=0.12$ ), and the survival of patients receiving antilymphocyte globulin/cyclosporin A was 


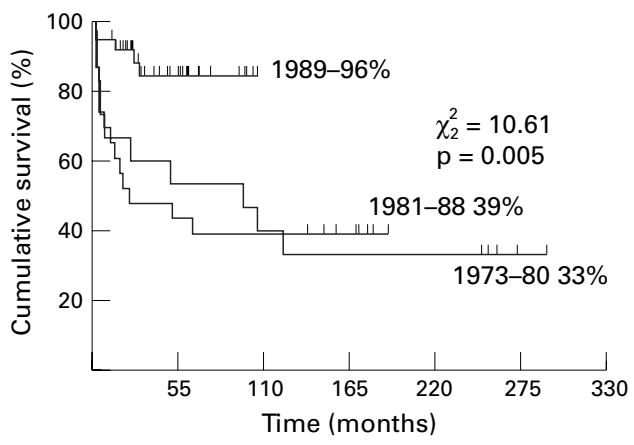

Figure 1 Overall survival of children treated for aplastic anaemia in three time periods.

better than that of those given antilymphocyte globulin alone $(100 \%$ v 52\%; $\mathrm{p}=0.01)$, although two children given antilymphocyte globulin/cyclosporin A required a bone marrow transplant because of treatment failure, and one further child has relapsed and received additional immunosuppressive treatment. The quality of responses in group B children appeared to be improved after antilymphocyte globulin/cyclosporin A (five complete responses, seven partial responses, and two no responses) compared with antilymphocyte globulin (five complete responses, one partial response, and five no responses), but this difference was not significant. Three patients with no response died without further specific treatment, one as a result of intracranial haemorrhage, and two because of infection (one as a result of disseminated aspergillosis and one as a result of staphylococcal septicaemia), and four received a bone marrow transplant (see below). Five of the 19 patients in group B who responded to immunosuppressive treatment subsequently relapsed-two of these patients received a bone marrow transplant, and two achieved a partial response and one a complete response with further immunosuppressive treatment. Minor cytopenia persists in 11 of the 18 patients in group B who survived without a bone marrow transplant; eight with thrombocytopenia (median platelet count, $93 \times 10^{9} / 1$; range, $\left.71-123\right)$ and five with neutropenia (median, $1.56 \times 10^{9} / 1$; range, 1.01-1.80), of whom two have both thrombocytopenia and neutropenia. No patient in either group developed a second malignancy or clonal disorder on long term follow up.

BONE MARROW TRANSPLANTATION

In group $\mathrm{A}$, there were five bone marrow transplant related deaths, including two patients with varicella infection following matched sibling donor bone marrow transplantation, and all three matched unrelated donor bone marrow transplants, as a result of failed engraftment. The 10 patients in group B who received primary treatment with a matched sibling donor bone marrow transplant are all clinically well, with total engraftment and normal blood counts. Three patients had acute skin GVHD (grade I, $\mathrm{n}=2$; grade II, $\mathrm{n}=1$ ), but no patient had chronic GVHD. One patient receiving a matched unrelated donor bone marrow transplant developed listeria meningi-

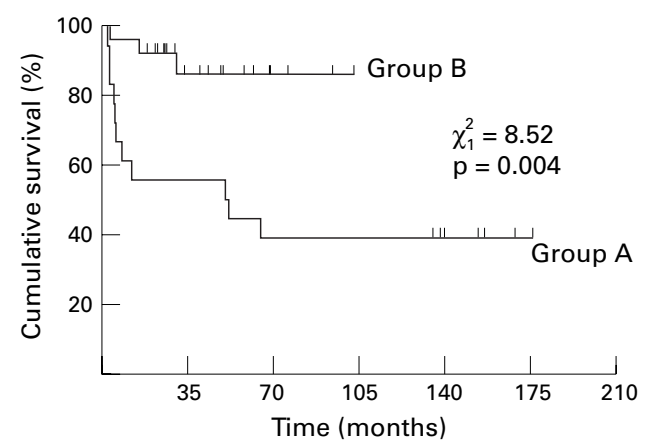

Figure 2 Overall survival of all children treated with antilymphocyte globulin. Three children in group $A$ and six in group $B$ received a bone marrow transplant after failure of primary immunosuppression.

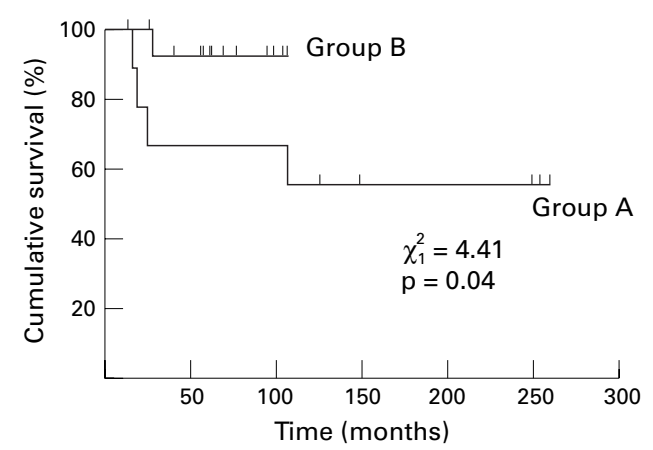

Figure 3 Overall survival of all children receiving a bone marrow transplant.

tis, and died nine months later. Four of the five children in group B surviving after bone marrow transplantation following failed immunosuppressive treatment show full engraftment, one with a mild persistent neutropenia $\left(1.50 \times 10^{9} / 1\right)$, and another with minimal thrombocytopenia $\left(130 \times 10^{9} / 1\right)$. One patient who received a matched sibling donor bone marrow transplant after failed immunosuppressive treatment developed late graft failure and required a further course of cyclosporin A after the bone marrow transplant. He has mild thrombocytopenia $\left(140 \times 10^{9} / 1\right)$ and neutropenia $\left(1.3 \times 10^{9} / 1\right)$ one year after cessation of cyclosporin A.

\section{Discussion}

Our findings confirm an improved prognosis for children with acquired AA treated either with immunosuppression or bone marrow transplantation. The most important changes to treatment during the study period have been the abandonment of androgens, the introduction of cyclosporin A to immunosuppressive regimens, and improved conditioning and GVHD prophylaxis for bone marrow transplantation. Other factors that might have contributed to improved survival include the availability of higher quality (particularly leucocyte depleted) blood products, developments in antimicrobial treatment and prophylaxis, refinements to air filtration in providing a protective environment for bone marrow transplantation, and the use of growth factors during both immunosuppressive treatment and bone marrow transplantation. ${ }^{3911}$ 
Allogeneic bone marrow transplantation from a matched sibling donor has been established as a superior treatment to immunosuppressive treatment regimens not containing cyclosporin $\mathrm{A}^{11-13}$ for young patients with severe AA. Most of the recent data indicating an increased survival following matched sibling donor bone marrow transplantation refer to the treatment of adults. The improved survival after matched sibling donor bone marrow transplantation for AA has been attributed to reduced mortality in the first three months after transplantation, achieved by advances in supportive care and GVHD prophylaxis, including cyclosporin $\mathrm{A} .{ }^{14}$ In our study, the better outcome for matched sibling donor bone marrow transplantation in group B was a result of a reduction in infective deaths.

Results for bone marrow transplantation after failed immunosuppressive treatment appeared to be much better in group B-group A patients who failed to respond or relapsed after immunosuppressive treatment had a dismal prognosis, even if a matched donor was available, whereas successful bone marrow transplantation was possible in five of six patients in group B. The risk of graft failure, responsible for the three matched unrelated donor bone marrow transplantation related deaths in group A, has been reduced by more intensive preparative regimens and better immunosuppression, ${ }^{3}$ and the improved outcome for matched unrelated donor bone marrow transplantation, also documented by others,${ }^{10}$ is encouraging for the treatment of children who fail immunosuppressive treatment.

The response to immunosuppressive treatment with antilymphocyte globulin alone was similar throughout both time periods, but the addition of cyclosporin $\mathrm{A}$ in patients from group B was associated with a better response and overall survival. However, this was not a randomised trial and other changes might have produced this benefit. The outcome for all patients receiving immunosuppressive treatment in group B (including 11 of 25 not receiving cyclosporin A) approached that for patients receiving bone marrow transplants (86\% v 93\% actuarial survival at eight years). Previous reports have shown that cyclosporin A produces a $20-40 \%$ increase in response rate compared with antilymphocyte globulin alone or in combination with androgens, ${ }^{7}$ and a recent multicentre trial of immunosuppressive treatment with combined antithymocyte globulin/cyclosporin $\mathrm{A}^{15}$ showed response rates similar to this study $(88 \%$ in children with moderate and severe AA). In the latter study, no benefit was obtained from a second course of ATG, although this was given early, within one month of initial treatment. The optimal scheduling of antilymphocyte globulin/ antithymocyte globulin and cyclosporin A remains controversial. Variations in the regimens reported, as well as differences in response criteria used make direct comparisons with the outcome of other studies difficult. In a retrospective, 12 year review of 27 AA patients, Lawlor and colleagues ${ }^{8}$ found similar actuarial survival for patients receiving bone marrow transplants and antithymocyte globulin/cyclosporin A, leading to a proposal that immunosuppressive treatment should be considered as initial treatment for all patients, regardless of the availability of a matched sibling donor. Mortality and toxicity from bone marrow transplantation in their study were high, possibly because of differences in GVHD prophylaxis and the use of HLA identical parental donors in two cases; in addition, the children receiving bone marrow transplantation were significantly older than those receiving immunosuppressive treatment, and it is premature to recommend the replacement of matched sibling donor bone marrow transplantation by immunosuppressive treatment for severely affected children.

While improved immunosuppressive treatment with antilymphocyte globulin and cyclosporin A might have reduced the survival advantage afforded by matched sibling donor bone marrow transplantation, several important disadvantages of immunosuppressive treatment remain. Haematological recovery when using immunosuppressive treatment is slower and/or less complete than for matched sibling donor bone marrow transplantation, resulting in a longer period of risk for bleeding, infection, and increased exposure to blood products. In our study, relapse occurred in about a quarter of patients, similar to other reports, although a high proportion responded to further immunosuppressive treatment, particularly when cyclosporin $\mathrm{A}$ is used. ${ }^{16}$ Late relapses are common and underline the need for adequate follow up periods when comparing treatment outcomes.

Following successful immunosuppressive treatment in adults, there is a significant risk of developing second tumours and clonal haematological diseases including myelodysplasia, acute myeloid leukaemia and paroxysmal nocturnal haemoglobinuria. ${ }^{16-18}$ No secondary haematological disorders have been identified in our patients followed clinically with regular blood counts and examination of the blood film, although more comprehensive assessments are currently underway. Although there is a paucity of data for children from other studies, clonal disorders and/or myelodysplasia appear less common following immunosuppressive treatment, but a high incidence was reported among children in Japan receiving immunosuppressive treatment including cyclosporin A combined with growth factors. ${ }^{19}$ In contrast with immunosuppressive treatment, the long term outcome after bone marrow transplantation for AA is excellent, ${ }^{18}{ }^{20}$ haematological reconstitution is usually rapid and sustained, with earlier resumption of a normal quality of life. The risk of second malignancy after bone marrow transplantation is largely a result of the use of irradiation, which is avoidable for children receiving matched sibling donor bone marrow transplant. ${ }^{13}$

Therefore, the improved survival for children with AA is multifactorial. Our findings confirm that where a compatible donor is available, matched sibling donor bone marrow 
transplantation is highly effective and remains the treatment of choice for severe $\mathrm{AA}$ in children, and that results of immunosuppressive treatment have improved greatly, confering a good outcome for most children who are treated. Matched unrelated donor bone marrow transplantation appears to be justified where immunosuppression has failed, but is associated with a higher risk and poorer outcome. The results of prospective multicentre trials with adequate follow up are awaited to determine the independent influence of growth factors on the prognosis for AA, and whether primary immunosuppressive treatment including cyclosporin $\mathrm{A}$ is as safe and effective as a matched sibling donor bone marrow transplant.

We thank Mr J McDonald-Clink for assistance in the statistical analysis.

1 Clausen N, Kreuger A, Salmi T, et al. Severe aplastic anaemia in the Nordic countries: a population based study of incidence, presentation, course and outcome. Arch Dis Child 1989;74:319-22.

2 Camitta BM, Storb R, Thomas ED. Aplastic anaemia; pathogenesis, diagnosis, treatment and prognosis. $\mathrm{N} \mathrm{Engl} \mathcal{F}$ Med 1982;306:712-18.

3 Young NS, Barrett AJ. The treatment of severe acquired aplastic anaemia. Blood 1995;85:3367-77.

4 Webb DK, Hann IM, Chessells JM. Acquired aplastic Webb DK, Hann IM, Chessells JM. Acquired aplastic 858-61.

5 Katsanis E, Ramsay NKC. Treatment of acquired severe aplastic anaemia. The American fournal of Pediatric Hematology/Oncology 1989;1:360-7.

6 Matloub YH, Bostrom B, Golembe B, Priest J, Ramsay NKC. Antithymocyte globulin, cyclosporin, and prednisolone for the treatment of severe aplastic anaemia in children. The American fournal of Pediatric Hematology/ Oncology 1994;16:104-6.

7 Rosenfeld SJ, Kimball J, Vining D, Young NS. Intensive immunosuppression with antithymocyte globulin and cyclosporine as treatment for acquired aplastic anemia. Blood 1995;85:3058-65.

8 Lawlor ER, Anderson RA, Davis JH, et al. Immunosuppressive therapy: a potential alternative to bone marrow trans- plantation as initial therapy for acquired severe aplastic anemia in childhood. F Pediatr Hematol Oncol 1997;19:11523

9 Bacigalupo A, Broccia G, Corda G, et al for the European Group for Blood and Marrow Transplantation (EBMT) Working Party on SAA. Antilymphocyte globulin, cyclosporin, and granulocyte colony-stimulating factor in patients with acquired severe aplastic anaemia (SAA): a pilot study of the EBMT SAA working party. Blood pilot study of the

10 Margolis D, Camitta B, Pietryga D, et al. Unrelated donor bone marrow transplantation to treat severe aplastic anaemia in children and young adults. $\mathrm{Br} \mathcal{F}$ Haematol 1996;94:65-72.

11 Werner EJ, Stout RD, Valdez LP, Harris RE. Immunosuppressive therapy versus bone marrow transplantation for children with aplastic anemia. Pediatrics 1989;83:61-5.

12 Halperin DS, Grisaru D, Freedman MH, Saunders EF. Severe acquired aplastic anemia in children: 11-year experience with bone marrow transplantation and immunosuppressive therapy. The American fournal of Pediatric Hematology/Oncology 1989;11:304-9.

13 Locasciulli A, Van't Veer L, Bacigalupo A, et al. Treatment with marrow transplantation or immunosuppression of childhood acquired severe aplastic anemia: a report from the EBMT SAA working party. Bone Marrow Transplant 1990;6:211-17.

14 Passweg JR, Socié G, Hinterberger W, et al. Bone marrow transplantation for severe aplastic anemia: has outcome improved? Blood 1997;90:858-64

15 Matloub YH, Smith C, Bostrom B, et al. One versus two courses of antithymocyte globulin for the treatment of severe aplastic anaemia. I Pediatr Hematol Oncol 1997;19: 110-14.

16 Rosenfeld SJ, Young NS. Aplastic anemia treated by immunosuppression is a chronic relapsing illness, but prognosis is unaffected by relapse. Blood 1997;90(suppl 1):435.

17 Socié G, Henry AM, Bacigalupo A, et al. Malignant tumors occurring after treatment of aplastic anemia. European bone marrow tansplantation-severe aplastic anemia working party. N Engl f Med 1993;329:1152.

18 Paquette RL, Tebyani N, Frane M, et al. Long-term outcome of aplastic anemia in adults treated with anti-thymocyte globulin: comparison with bone marrow transplantation. Blood 1995;85:283-90.

19 Ohara A, Kojima S, Hamajima N, et al. Myelodysplastic syndrome and acute myelogenous leukemia as a late clonal complication in children with acquired aplastic anemia. Blood 1997;90:1009-13.

20 Reiter E, Keil F, Brugger S, et al. Excellent long-term survival after allogeneic marrow transplantation in patients with severe aplastic anaemia. Bone Marrow Transplant 1997;19:1191-6. 